\title{
Septic Shock Induced by Vibrio Vulnificus in Northern Poland, a Case Report
}

\author{
Bogusz Jan Aksak-Wąs (iD \\ Agnieszka Ripa ${ }^{2}$ \\ Paweł Szakoła ${ }^{3}$ \\ Karolina Horbacka ${ }^{3}$ \\ Jolanta Niścigorska-Olsen ${ }^{2}$ \\ Magdalena Witak-Jędra ${ }^{2}$ \\ Małgorzata Zając- \\ Marczewska ${ }^{2}$ \\ Malwina Karasińska- \\ Cieślak ${ }^{2}$ \\ Jacek Kot ${ }^{4}$ \\ Miłosz Parczewski \\ 'Department of Infectious, Tropical \\ Diseases and Immune Deficiency, \\ Pomeranian Medical University in \\ Szczecin, Szczecin, Poland; ${ }^{2}$ Department \\ of Infectious, Tropical Diseases and \\ Immune Deficiency, Provincial Hospital, \\ Szczecin, Poland; ${ }^{3}$ Department of \\ General and Transplant Surgery, \\ Department of Vascular Surgery, \\ Provincial Hospital, Szczecin, Poland; \\ ${ }^{4}$ National Center for Hyperbaric \\ Medicine, University Center for Maritime \\ and Tropical Medicine in Gdynia, Medical \\ University of Gdansk, Gdansk, Poland
}

Correspondence: Bogusz Jan Aksak-Wąs Email bogusz.aw@gmail.com

\begin{abstract}
Introduction: Vibrio vulnificus infections are a growing problem worldwide. In recent years, infections with this bacteria have been reported in Central Europe, especially in the German Baltic coast but also in France and Italy. Climate warming causes the sea temperature to increase every year, which translates to an increased risk of infections from the Vibrio group. Most of these are mild and present as wound infections, but some patients develop life-threatening sepsis from either ingestion of infected mollusks or wound lesions that develop into generalized infections. Illness may be associated with necrotizing fasciitis and may require several weeks of therapy, often based on a surgical operation, demarcation of necrosis or limb amputation. A case such as the one described in this manuscript has not been previously described in Poland and demonstrates the need for a multidisciplinary approach to infection with Vibrio vulnificus.
\end{abstract}

Case Presentation: A 68-year-old patient was pricked with an unknown object in the side of a lower limb during his stay at the Polish seaside. He developed a life-threatening infection in the form of severe sepsis with multiple organ failure. He required broadspectrum antibiotic therapy, and after obtaining results for Vibrio vulnificus targeted therapy, a surgical operation with skin lesion decompression and fasciotomy was performed. Finally, hyperbaric chamber therapy was given. The patient's general condition improved, and local changes and his vital parameters stabilized.

Conclusion: Vibrio vulnificus infection may be confused with other causes of skin and subcutaneous tissue infection, although it requires a different approach and different targeted antibiotic therapies. This infection may take the form of a life-threatening disease requiring a multidisciplinary approach.

Keywords: case report, Vibrio vulnificus, sepsis shock, crustaceans, hyperbaric chamber

\section{Introduction}

Vibrio vulnificus is a ubiquitous, gram-negative, comma-shaped bacterium typically found in the Gulf of Mexico but also Massachusetts Bay, areas near Washington state, and far-east Asia, ${ }^{1}$ coastal areas and estuaries. It lives in brackish water (in river estuaries, where it is possible to mix fresh and salt water). It prefers a tropical and subtropical climate with a water temperature above $18^{\circ} \mathrm{C}$, but it has also been isolated in waters with a lower average temperature.

Infection with Vibrio vulnificus is a significant cause of mortality from seafood in the US. ${ }^{1}$ In coastal areas of the US, the incidence is $0.5 / 100,000$ people. Since 2003, isolated cases of infections with this pathogen have been reported on the German Baltic coast. The source of infection is filter mollusks, primarily oysters. ${ }^{2}$ Infection is possible 
through ingestion of contaminated food and from the shells or spicules of infected mollusks, fishes, or crustacea.

Vibrio vulnificus can cause infection of wounds after entry into the body via this route or primary sepsis ${ }^{3}$ (mainly among patients who eat infected mollusks), with a mortality of over $90 \%$, especially in patients presenting for care with hypotension and in patients with hepatic insufficiency. 3,4

Although Vibrio vulnificus may exist in the Baltic Sea, no other case of this kind has been reported previously in Poland, particularly one requiring a multidisciplinary, conservative and surgical, approach.

\section{Case Presentation}

The patient was a 68-year-old pensioner, living in Germany since 1989.

His history of comorbidities was as follows: arterial hypertension and permanent atrial fibrillation. Due to chronic diseases, the patient was taking fenprocoumon, simvastatin, and torsemide on a regular basis. The patient was vaccinated during his stay in Germany with 2 doses of the SARS-COV-2 vaccine.

\section{Medical History}

From July 11, 2021, the patient stayed in Niechorze (West Pomeranian Voivodeship, Poland) on holiday. On July 14, while swimming in the sea, he felt a prick on the side of his left leg (small, 2-3 mm diameter). The stab was minor but painful. He disinfected the wound but did not cover it with a dressing. In the following days, the wound was covered with a scab. Despite its presence, he bathed in the sea the following days. In the interview, he denied eating raw fish or seafood, including mollusks and crustaceans. He ate fried fish like the rest of the family. No one around him was as sick as he was. On July 18, at approximately 11 p.m., he noticed increasing swelling and redness of the left lower leg. At approximately 5 a.m. on the following day, he was taken to the Emergency Department of a nearby district hospital, where a $10 \mathrm{~cm}$ lesion was found on the lateral side of the left lower leg with an area of necrosis with serous blisters that were strongly palpable. He had a normal blood pressure (115/ $67 \mathrm{mmHg}$ ), and his heart rate was 90/min. Amoxicillin with clavulanic acid and clindamycin and analgesics were recommended, and the patient was referred to the Department of Infectious Diseases provincial hospital. The patient was sent to the local department around noon on the same day. On the way to the local ward, the patient was vomiting, and during his stay in the emergency room, he reported nausea. On admission, he reported pain in the lower leg with an NPRS of 10; no improvement from the ketoprofen administered during his stay in the district hospital was observed, and chest tightness was reported. His BP was $84 / 45 \mathrm{mmHg}$, indicating hypotension, with a heart rate of approximately $62 / \mathrm{min}$ that was completely irregular. The following conditions were also observed: saturation with atmospheric oxygen $100 \%$, respiratory rate $12 /$ minute, and body temperature (in non-contact measurement) 35.9 degrees C. The GCS score was 15, and the qSOFA score was 1 . During the physical examination in the local ward, the patient was in a mediocre state. The patient was able to think logically and had a balanced mental state. The skin was subjectively clean, dry, and without cyanosis. The patient was overweight (height: $168 \mathrm{~cm}$; weight: $78 \mathrm{~kg}$; BMI: 27.64; BSA: $1.88 \mathrm{~m} 2$ ). On the left leg, there was significant swelling, redness, and numerous large blisters filled with serous bloody contents, some of which ruptured and had oozing discharge (Figure 1). On the other lower limb, there were trophic changes. A scar formed after insertion of the nerve stimulator in the right subclavian area. Scars

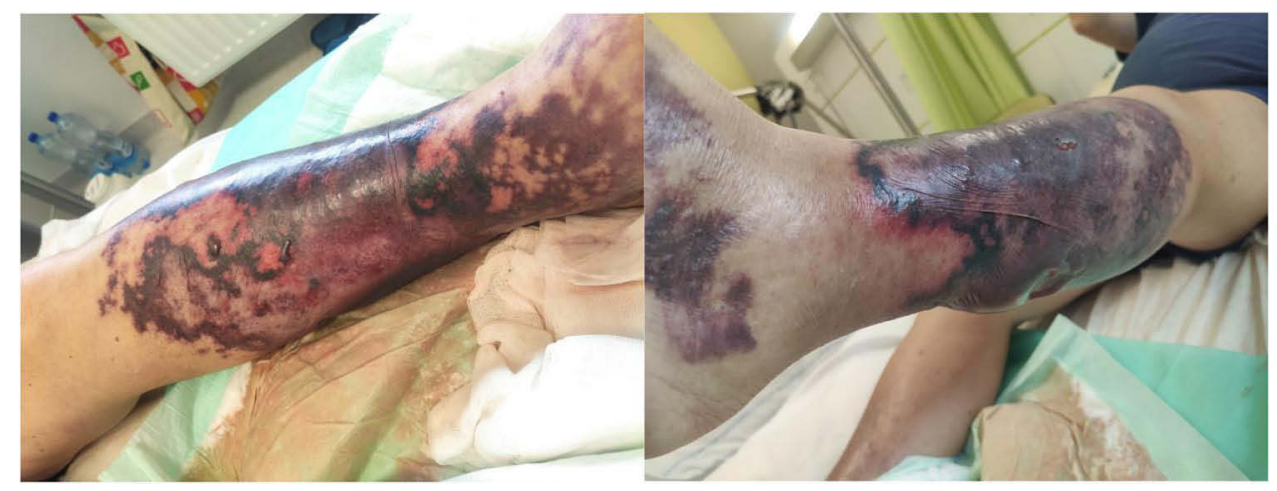

Figure I Image of the lower limb before therapy. 
were also noted on the forearm and right hand and in the lumbar region of the spine. The oral mucosa was moist, no raids were present, the throat was pink, and the palatine tonsils were not enlarged. Peripheral lymph nodes were not enlarged, including those in the area of the skin lesions. The lungs displayed overt convexity, a vesicular murmur, and crackles at the base. Heart tones were clear. The abdomen was soft and pain-free, without pathological resistance. The liver and spleen were not enlarged, peritoneal symptoms were negative, and the patient was negative for Chełmoński and for Goldflam symptoms on both sides. He was also negative for meningeal symptoms.

\section{Laboratory Values at Presentation}

The laboratory values for the patient were as follows: normoleukocytosis, CRP $30.33 \mathrm{mg} / \mathrm{l}$; procalcitonin 15.80 $\mathrm{ng} / \mathrm{mL}$; and interleukin $6>5000 \mathrm{pg} / \mathrm{mL}$. Alanine aminotransferase (ALAT) and aspartate (ASPAT) levels were normal; gamma-glutamyltranspeptidase (GGTP) levels were slightly elevated; total bilirubin was $1.42 \mathrm{mg} / \mathrm{dl}$; and INR was 4.33. Plasma albumin levels were normal. The plasma creatinine level was $1.73 \mathrm{mg} / \mathrm{dl}$. Hypogammaglobulinemia was present with an IgG level of $0.70 \mathrm{~g} / \mathrm{dl}$; the lactate level was $11.68 \mathrm{mmol} / 1$.

In the local department, broad-spectrum empirical antibiotic therapy with cephalosporin of the third generation (ceftriaxone) and intravenous clindamycin was initially introduced. Due to the elevated levels of renal creatinine, intensive fluid therapy was administered, although the patient did not achieve either blood pressure normalization (systolic blood pressure $<90 \mathrm{mmHg}$, MAP $<65$ ) or normalization of renal parameters (increase in renal creatinine level to $3.68 \mathrm{mg} / \mathrm{dl}$ ). Despite the unsatisfactory blood pressure levels, it was decided to use forced diuresis with little effect (diuresis approximately $1000 \mathrm{~mL} /$ day). Ionic deficiencies were supplemented, and the level of kalaemia quickly normalized. Due to the coagulation disorders caused by the intake of vitamin $\mathrm{K}$ antagonists by the patient, supplements of this vitamin were given. Due to the severe pain in the limb, the patient required opioids, which had a good effect, and the pain was finally relieved to an NPRS score of 1 .

On the first day of hospitalization, information was obtained from the microbiological laboratory about a positive result for Vibrio vulnificus in blood cultures (MALDI-TOF-mass spectrometry). An antibiogram was performed from blood preparations and wound swabs (a total of 4 blood cultures and a bacteriological wound swab).

Susceptibility was determined with disc-diffusion method according to the recommendations of CLSI, M45, 3rd edition.

The sensitivity of the cultured microbes to antibiotics was as follows:

Cefotaxime $1=\mathrm{S}\{\mathrm{MIC}=0.032\}$

Ceftazidime $1=\mathrm{S}\{\mathrm{MIC}=0.75\}$

Imipenem $1=\mathrm{S}\{\mathrm{MIC}=0.094\}$

Meropenem $1=\mathrm{S}\{\mathrm{MIC}=0.012\}$

Ciprofloxacin $1=\mathrm{S}\{\mathrm{MIC}=0.016\}$

Levofloxacin $1=\mathrm{S}\{\mathrm{MIC}=0.016\}$

Tetracycline $1=\mathrm{S}$

Before obtaining the antibiogram, clindamycin was discontinued, and doxycycline and intravenous ciprofloxacin were empirically added to the therapy (doses adjusted to renal function). The patient's parenteral irrigation was continued. There was a decrease in the number of platelets to 66.00 thousand/L norm: [140.00-420.00], and a slight elevation of hepatic parameters: ASPAT to $71 \mathrm{U} / \mathrm{L}$ norm: $[<40]$; ALAT up to $66 \mathrm{U} / \mathrm{L}$ standard: $[<41]$. Despite the implementation of antibiotic therapy, an increase in inflammatory parameters was observed, with CRP increasing to $255.80 \mathrm{mg} / \mathrm{l}$ and procalcitonin to $95.50 \mathrm{ng} / \mathrm{mL}$. A very slow decrease in the level of INR was obtained, so treatment with low molecular weight heparin was initiated.

On the second day of stay, due to increasing inflammatory parameters and the patient's deteriorating clinical picture (increasing limb swelling, increasing skin necrotic changes), the patient was transferred to the surgery department of the local hospital for fasciotomy. CT examination of the lower left limb was performed, excluding features of necrotizing fasciitis (Figure 2). During his stay in the surgical ward, the patient required oxygen therapy and periodic infusion of norepinephrine to obtain an MAP over 65 , and the patient was in fairly good general condition, with a GCS score of 15.

On July 21, 2021, an incision was made, and decompression of the skin and subcutaneous tissue of the left leg and a fasciotomy were performed (Figure 3). During hospitalization, targeted antibiotic therapy, fluid therapy and forced diuresis were administered. A decrease in inflammatory and renal parameters was observed. The patient qualified for treatment with hyperbaric oxygen. On July 22, he was transferred to a hyperbaric center in Gdynia (Pomeranian Voivodeship, Poland) for further general, surgical and hyperbaric treatment. 


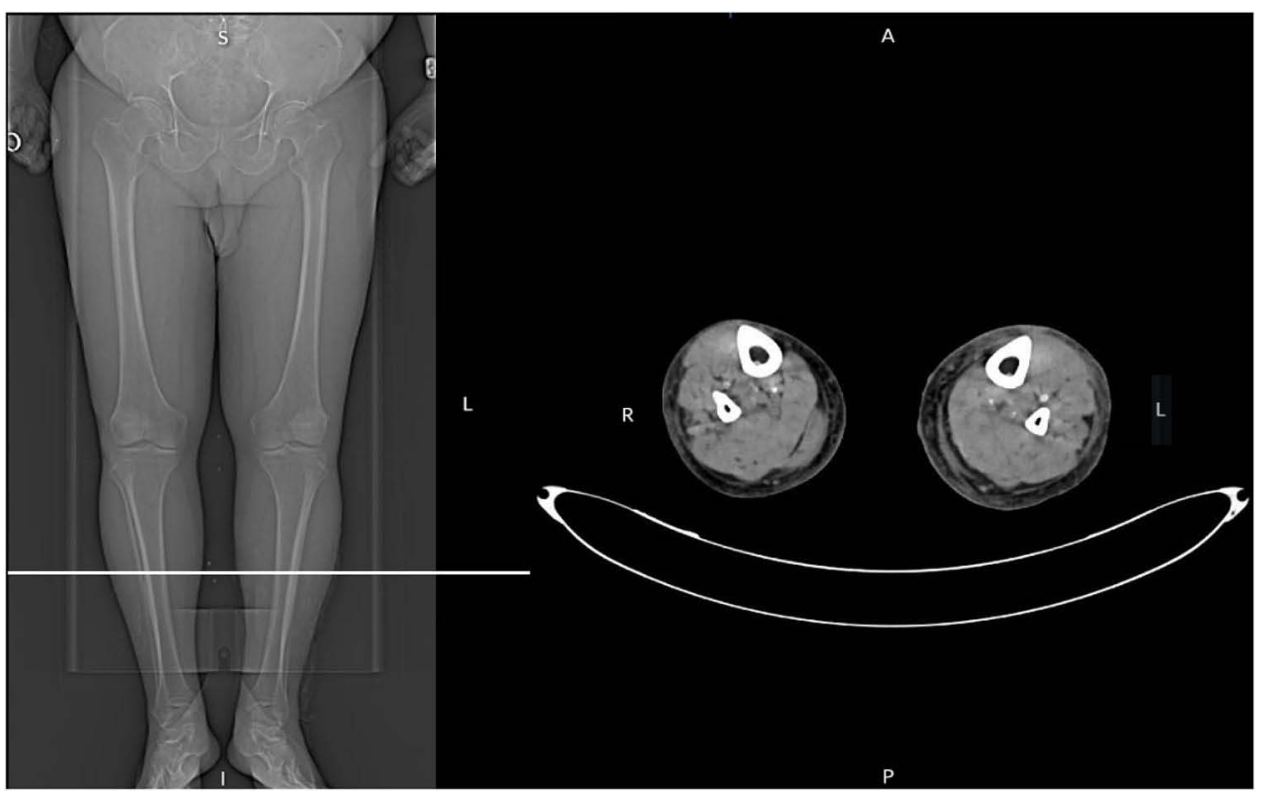

Figure 2 Computed tomography image of the altered limb.

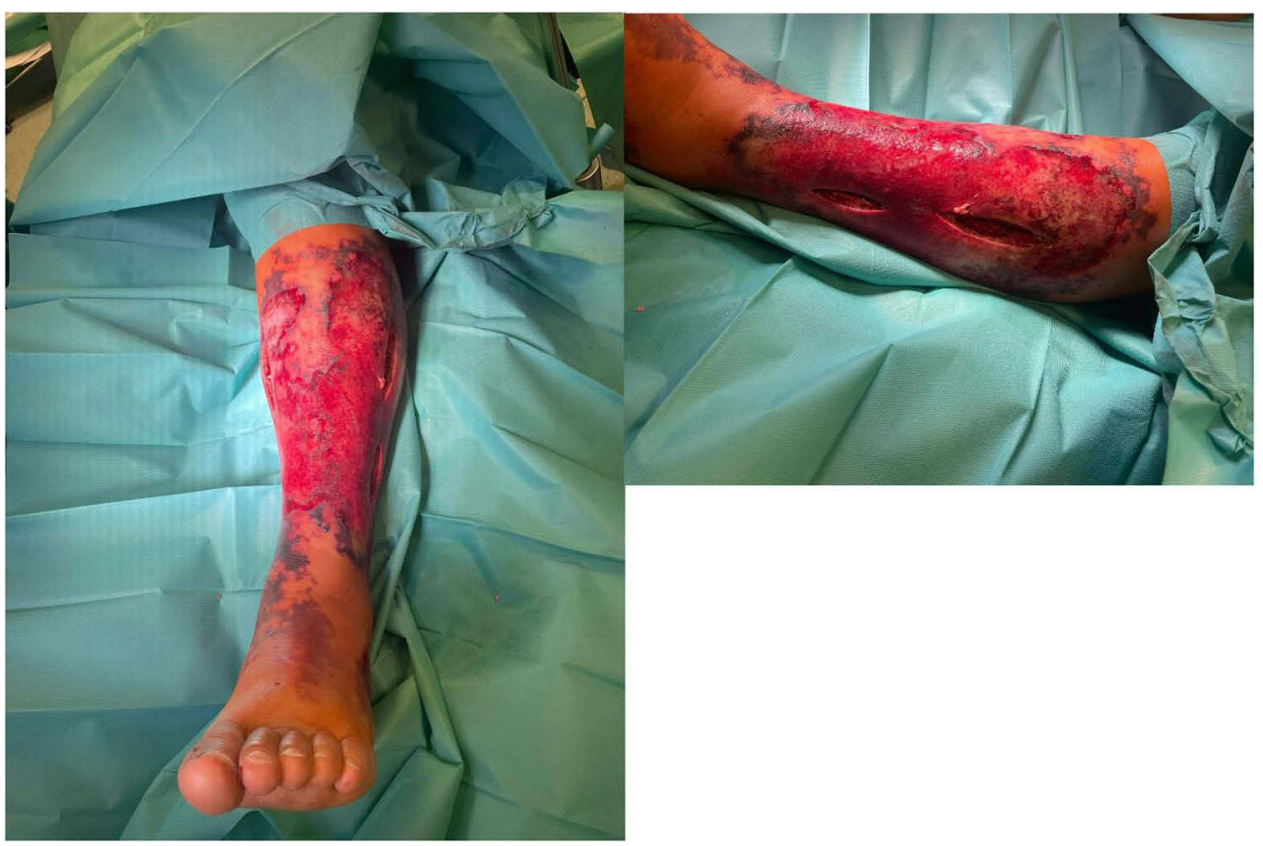

Figure 3 Image of the lower limb during surgery.

The patient underwent hyperbaric oxygen therapy (HBOT) sessions twice a day in the multiplace hyperbaric chamber, dressings of the lower limb were changed daily, and antibiotic therapy, symptomatic treatment, and pain relief were administered. During this stay, a single increase in body temperature to $37.8^{\circ} \mathrm{C}$ was observed without general symptoms. At the local center, the patient was treated with a total of 10 sessions of HBOT at 2.5 ATA for $60 \mathrm{~min}$ with $100 \% \mathrm{O} 2$.

On July 28, the patient was returned to the local observation and infectious disease ward (Szczecin, Zachodniopomorskie Province, Poland) after hyperbaric treatment in accordance with the usual practice in Poland, in order to continue wound treatment and antibiotic therapy. Patient was complaining of 
only slight pain in the lower limb during manipulations and slight pain when walking. On admission, on the skin of the left shank, 4 decompression incisions were found; some of the wounds were covered with fibrin, and the superficial layer of the epidermis was bare, with the dermis shown. Due to significant local improvement and good sensitivity of found microorganism, no control swabs were collected. Clearly marked cellulitis was present on the inside of the thigh up to the left groin (Figure 4). In addition, small foci of herpes labialis were visible on the upper lip. The following conditions were observed: GCS: 15 , qSOFA: 0 , heart rate $65 / \mathrm{min}$, and BP: $150 / 80 \mathrm{mmHg}$. His atmospheric oxygen saturation was $96 \%$, and his temperature was 36.7 degrees Celsius.

\section{Laboratory Values During the Second Stay}

The laboratory values of the patient were as follows: WBC indicated normoleukocytosis; number of platelets 149.00 thousand $/ \mu \mathrm{L}$; CRP $79.85 \mathrm{mg} / \mathrm{L}$; procalcitonin $0.45 \mathrm{ng} / \mathrm{mL}$; interleukin $646.5 \mathrm{pg} / \mathrm{mL}$; creatinine $0.78 \mathrm{mg} / \mathrm{dL}$; INR 1.81, ALAT $50 \mathrm{U} / \mathrm{L}$; and ASPAT $53 \mathrm{U} / \mathrm{L}$.

The patient's treatment included the following: thirdgeneration cephalosporin (12 days of therapy in total), doxycycline (11 days of therapy in total), and ciprofloxacin (10 days of therapy in total). The patient tolerated the treatment without any complications, the drugs were administered by the hospital staff, and the adherence was $100 \%$.
At his own request, the patient was transferred to hospital with a plastic surgery department in his country of residence.

\section{Discussion and Conclusion}

Infection with Vibrio vulnificus has thus far been associated with warmer climates, mainly in the United States ${ }^{4}$ but also in Southern Europe and Asia. ${ }^{5,6}$ Due to global warming, it is a growing problem in the German coastal zone of the Baltic Sea. ${ }^{7}$ Germany shares this coastal zone with Poland, so it was only a matter of time before the first cases of these infections in Poland were confirmed, although at the time of writing this manuscript, no descriptions of infections with this bacterium had been found in widely available periodicals.

Infection with Vibrio vulnificus can take the form of a mild wound infection or severe primary sepsis, usually caused by the consumption of infected oysters and other filter mollusks. ${ }^{8}$ The infection mainly affects men $(>90 \%$ of cases) and people over 40 years of age $(>85 \%$ of cases), ${ }^{9}$ which is consistent with the characteristics of the described case. The described patient developed not only severe wound infection but also severe sepsis with multiple organ failure. Mortality in severe courses of the disease reaches $90 \%$, with immunodeficiency, diabetes mellitus and liver disease exacerbating the course of infection, ${ }^{3,4,10}$ especially in patients presenting for care with low blood pressure. ${ }^{3,4,10,11}$ The patient in the current
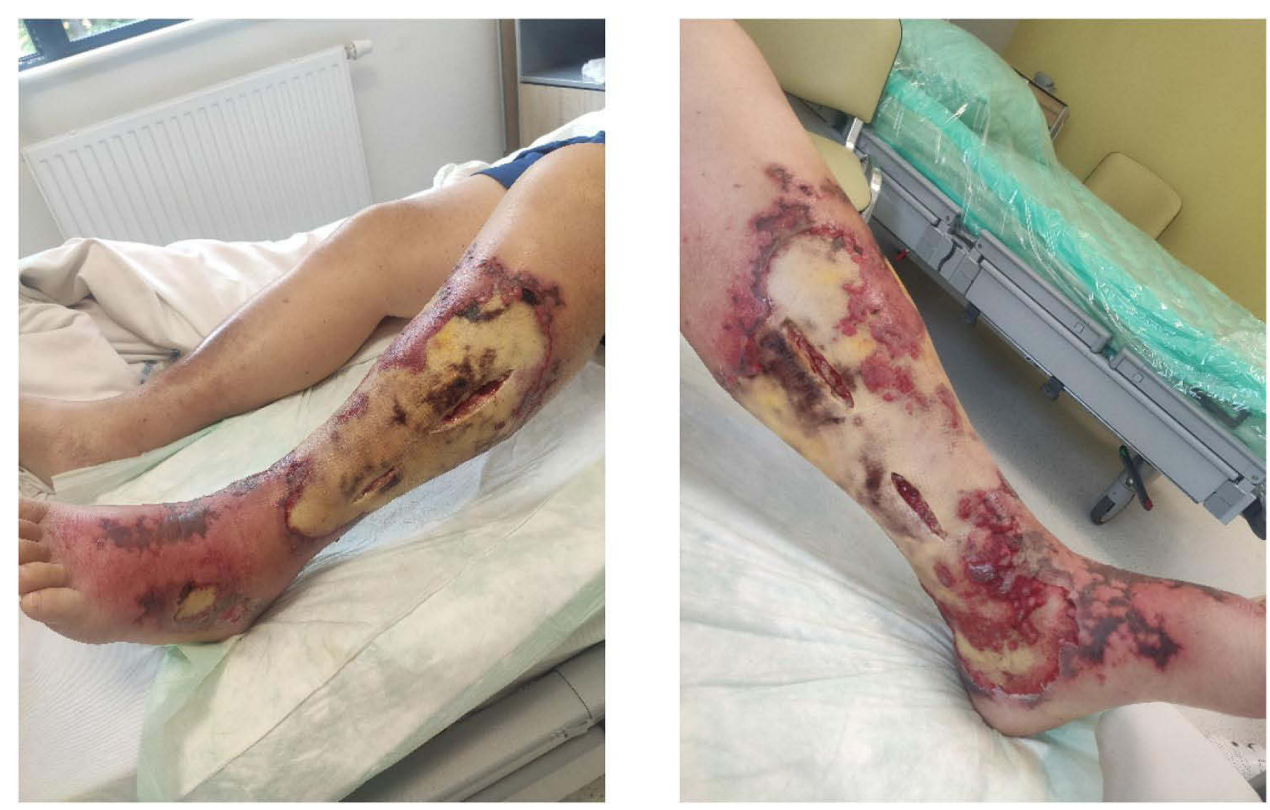

Figure 4 Image of the lower limb after returning from treatment in the hyperbaric chamber. 
case report did not have comorbid immunodeficiency or liver disease, although the baseline INR levels in the laboratory analysis were elevated due to the use of vitamin $\mathrm{K}$ antagonists (because of permanent atrial fibrillation). However, the patient came to the hospital and presented persistent hypotension during hospitalization, despite intensive fluid therapy with crystalloids requiring the use of pressor amines at some point, which is a very poor prognostic predictor.

Virulence of $V$. vulnificus has been associated with couple of potential factors: for example, production of an anti-phagocytic capsule, ${ }^{12}$ MARTX and other toxins, ${ }^{13}$ and iron acquisition systems. ${ }^{14} \mathrm{~V}$. vulnificus contains a lipopolysaccharide (LPS), which may directly trigger some cytokine responses, influencing occurrence of the shock syndrome. ${ }^{15}$ This bacteria may produce a variety of toxins cytolysin/hemolysin VvhA, and MARTX (multifunctional autoprocessing repeats-in-toxins), which could be a main factor of bacterial dissemination from the intestine with following sepsis. ${ }^{16}$

Vibrio vulnificus are usually susceptible to most antibiotics. ${ }^{17}$ However, in recent decades, massive overuse of antibiotics has led to increasing antibiotic resistance in numerous strains of bacteria. ${ }^{18}$ Vibrio vulnificus found in seafood are exhibiting resistance to multiple antibiotics due to the environmental misuse. ${ }^{19}$ Usually susceptible Vibrio vulnificus resistance toward common antibiotics has reached alarming levels in many countries. ${ }^{20}$

Earlier analyses indicated a combination of thirdgeneration cephalosporin antibiotics and either ciprofloxacin or doxycycline as a therapy to reduce mortality in patients, ${ }^{21}$ which was also applied in this situation. Numerous publications have indicated the need for rapid surgical therapy, especially among patients developing necrotizing fasciitis, ${ }^{22}$ even within 12 hours of hospital admission. Approximately $10 \%$ of patients require limb amputation in the course of the disease. ${ }^{4}$ Although the described patient did not develop necrotizing fasciitis, he was transferred to the surgical ward for decompression within 48 hours of reporting to the hospital, which improved his prognosis and further healing of skin lesions.

Some reports have indicated the use of hyperbaric oxygen or ultraviolet light to improve survival in patients infected with Vibrio vulnificus. ${ }^{23}$ It is indicated that bacteria are unable to repair DNA damage associated with the use of high-pressure oxygen. In the present case, a significant local improvement was also observed after the use of HBOT in the present patient.
Vibrio vulnificus infection may be confused with other causes of skin and subcutaneous tissue infection, although it requires a different approach and different targeted antibiotic therapies. This infection may take the form of a lifethreatening disease requiring a multidisciplinary approach, including specific targeted antibiotic therapy, intensive treatment of the symptoms of shock and multiorgan dysfunction, rapid surgical intervention and additional methods such as hyperbaric oxygen treatment.

\section{Abbreviations}

US, United States of America; SARS-COV-2, severe acute respiratory syndrome coronavirus-2; $\mathrm{C} 1,1$ st cervical vertebrae; NPRS, numeric pain rating scale; BP, blood pressure; GCS, Glasgow coma scale; qSOFA, quick SOFA scale; MAP, mean arterial pressure; CLSI, Clinical Laboratory Standards Institute; CT, computed tomography; HBOT, hyperbaric oxygen therapy; ATA, 1 atmosphere absolute.

\section{Consent for Publication}

Patient's written, informed consent for publication included case details as well as publication of images.

\section{Funding}

There is no funding to report.

\section{Disclosure}

The authors report no conflicts of interest in this work.

\section{References}

1. Morris JG, Black RE. Cholera and other vibrioses in the United States. $N \quad$ Engl $J \quad$ Med. 1985;312(6):343-350. doi:10.1056/ nejm198502073120604

2. Daniels NA. Vibrio vulnificus oysters: pearls and perils. Clin Infect Dis. 2011;52(6):788-792. doi:10.1093/cid/ciq251

3. Blake PA, Merson MH, Weaver RE, Hollis DG, Heublein PC. Disease caused by a marine vibrio: clinical characteristics and epidemiology. N Engl J Med. 1979;300(1):1-5. doi:10.1056/nejm197901043000101

4. Dechet A, Yu P, Koram N, Painter J. Nonfoodborne vibrio infections: an important cause of morbidity and mortality in the United States, 1997-2006. Clin Infect Dis. 2008;46(7):970-976. doi:10.1086/529148

5. Lorenzoni G, Tedde G, Mara L, et al. Presence, seasonal distribution and biomolecular characterization of Vibrio parahaemolyticus and Vibrio vulnificus in shellfish harvested and marketed in Sardinia (Italy) between 2017 and 2018. J Food Prot. 2021. doi:10.4315/JFP21-059

6. Hsueh PR, Lin CY, Tang HJ, et al. Vibrio vulnificus in Taiwan. Emerg Infect Dis. 2004;10(8):1363-1368. doi:10.3201/EID1008.040047

7. Metelmann C, Metelmann B, Gründling M, Hahnenkamp K, Hauk G, Scheer C. [Vibrio vulnificus, an increasing threat of sepsis in Germany?]. Der Anaesthesist. 2020;69(9):672-678. German. doi:10.1007/S00101-020-00811-9 
8. Bross MH, Soch K, Morales R, Mitchell RB. Vibrio vulnificus infection: diagnosis and treatment. Vol 76; 2007. Available from: www.aafp.org/afp. Accessed May 24, 2021.

9. Baker-Austin C, Oliver JD. Vibrio vulnificus. Trends Microbiol. 2020;28(1):81-82. doi:10.1016/J.TIM.2019.08.006

10. Tacket CO, Brenner F, Blake PA. Clinical features and an epidemiological study of Vibrio vulnificus infections. J Infect Dis. 1984;149 (4):558-561. doi:10.1093/INFDIS/149.4.558

11. Liu JW, Lee IK, Tang HJ, et al. Prognostic factors and antibiotics in Vibrio vulnificus septicemia. Arch Intern Med. 2006;166 (19):2117-2123. doi:10.1001/archinte.166.19.2117

12. Wright AC, Simpson LM, Oliver JD, Morris JG. Phenotypic evaluation of acapsular transposon mutants of Vibrio vulnificus. Infect Immun. 1990;58(6):1769-1773. doi:10.1128/IAI.58.6.17691773.1990

13. Chung K-J, Cho E-J, Kim M. RtxA1-induced expression of the small GTPase Rac2 plays a key role in the pathogenicity of Vibrio vulnificus. J Infect Dis. 2010;201(1):97-105. doi:10.1086/648612

14. Brennt CE, Wright AC, Dutta SK, Morris JG. Growth of Vibrio vulnificus in serum from alcoholics: association with high transferrin iron saturation. J Infect Dis. 1991;164(5):1030-1032. doi:10.1093/ INFDIS/164.5.1030

15. Powell JL, Wright AC, Wasserman SS, Hone DM, Morris JG. Release of tumor necrosis factor alpha in response to Vibrio vulnificus capsular polysaccharide in in vivo and in vitro models. Infect Immun. 1997;65(9):3713-3718. doi:10.1128/IAI.65.9.37133718.1997

16. Baker-Austin C, Oliver JD. Vibrio vulnificus: new insights into a deadly opportunistic pathogen. Environ Microbiol. 2018;20 (2):423-430. doi:10.1111/1462-2920.13955
17. Oliver JD. Vibrio vulnificus. Biol Vibrios. 2014;349-366. doi:10.1128/9781555815714.CH25

18. Cabello FC. Heavy use of prophylactic antibiotics in aquaculture: a growing problem for human and animal health and for the environment. Environ Microbiol. 2006;8(7):1137-1144. doi:10.1111/ J.1462-2920.2006.01054.X

19. Elmahdi S, DaSilva LV, Parveen S. Antibiotic resistance of Vibrio parahaemolyticus and Vibrio vulnificus in various countries: a review. Food Microbiol. 2016;57:128-134. doi:10.1016/J. FM.2016.02.008

20. Heng S-P, Letchumanan V, Deng C-Y, et al. Vibrio vulnificus: an environmental and clinical burden. Front Microbiol. 2017;8:997. doi:10.3389/FMICB.2017.00997

21. Kim SE, Shin SU, Oh TH, et al. Outcomes of third-generation cephalosporin plus ciprofloxacin or doxycycline therapy in patients with Vibrio vulnificus septicemia: a propensity score-matched analysis. PLoS Negl Trop Dis. 2019;13(6). doi:10.1371/JOURNAL. PNTD.0007478

22. Chao W-N, Tsai C-F, Chang H-R, et al. Impact of timing of surgery on outcome of Vibrio vulnificus-related necrotizing fasciitis. Am J Surg. 2013;206(1):32-39. doi:10.1016/J.AMJSURG.2012.08.008

23. Tamura T, Iida K, Saito M, Shiota S, Nakayama H, Yoshida S. Effect of hyperbaric oxygen on Vibrio vulnificus and murine infection caused by it. Microbiol Immunol. 2012;56(10):673-679. doi:10.1111/J.1348-0421.2012.00491.X
Infection and Drug Resistance

\section{Publish your work in this journal}

Infection and Drug Resistance is an international, peer-reviewed openaccess journal that focuses on the optimal treatment of infection (bacterial, fungal and viral) and the development and institution of preventive strategies to minimize the development and spread of resistance. The journal is specifically concerned with the epidemiology of
Dovepress

antibiotic resistance and the mechanisms of resistance development and diffusion in both hospitals and the community. The manuscript management system is completely online and includes a very quick and fair peerreview system, which is all easy to use. Visit http://www.dovepress.com/ testimonials.php to read real quotes from published authors. 